\title{
The Feasibility Study for Multigeometries Identification of Uranium Components Using PCA-LSSVM Based on Correlation Measurements
}

\author{
Mi Zhou, ${ }^{1,2}$ Peng Feng $\left(\mathbb{D},{ }^{1,3}\right.$ Yixin Liu $\mathbb{D},{ }^{4}$ and Biao Wei $\mathbb{D}^{1}$ \\ ${ }^{1}$ Key Laboratory of Optoelectronic Technology and System, Ministry of Education, Chongqing University, Chongqing 400044, China \\ ${ }^{2}$ School of Science, Chongqing University of Technology, Chongqing 400054, China \\ ${ }^{3}$ Collaborative Innovation Center for Brain Science, Chongqing University, Chongqing 400044, China \\ ${ }^{4}$ Department of Engineering Physics, Tsinghua University, Beijing 100084, China
}

Correspondence should be addressed to Peng Feng; coe-fp@cqu.edu.cn and Biao Wei; weibiao@cqu.edu.cn

Received 7 December 2017; Accepted 24 January 2018; Published 1 March 2018

Academic Editor: Arkady Serikov

Copyright (C) 2018 Mi Zhou et al. This is an open access article distributed under the Creative Commons Attribution License, which permits unrestricted use, distribution, and reproduction in any medium, provided the original work is properly cited.

\begin{abstract}
The geometry of uranium components is one of the key characteristics and strictly confidential. The geometry identification of metal uranium components was studied using ${ }^{252} \mathrm{Cf}$ source-driven correlation measurement method. For the 3 uranium samples with the same mass and enrichment, there are subtle differences in neutron signals. Even worse, the correlation functions were disturbed by scatter neutrons and include "accidental" coincidence, which is not conductive to the geometry identification. In this paper, we proposed an identification method combining principal component analysis and least-square support vector machine (PCA-LSSVM). The results based on PCA-LSSVM showed that the training precision was $100 \%$ and the test precision was $95.83 \%$ of the identification model. The total precision of the identification model was $98.41 \%$, which indicated that the identification model was an effective way to identify the geometry properties with the correlation functions.
\end{abstract}

\section{Introduction}

The geometry of uranium components is one of the key characteristics and strictly confidential [1,2]. As for uranium components, they are very difficult to be detected in passive measurements because of the low neutron yield and the weak energy of gamma ray. Uranium components can be shielded easily. Hence, the active method is considered and widely used.

Correlation measurements used for in the field of nuclear material identification have been developed in recent decades [3-11]. In active measurements, ${ }^{252} \mathrm{Cf}$ sources-driven correlation measurement method is a popular one $[12,13]$. Based on this method, ORNL (Oak Ridge National Laboratory) had designed a nuclear material identification system (NMIS) aiming to verify the fissile material [14-16]. This method has been used successfully in the past to identify various uranium components, but nearly all of them focused on mass and enrichment [7, 17-19]. Indeed, geometric difference exists practically, but few studies have been done.
In this paper, the research work of the geometry identification to the metal uranium components has been conducted. The uranium components have the same mass, enrichment, and different geometry. Just because the uranium components only have geometric difference, the geometry identification is very difficult by the correlation functions simply. By analyzing the features and differences of the correlation functions, we proposed an identification method combining principal component analysis and least-square support vector machine (PCA-LSSVM) $[20,21]$ to build an identification model, and the uranium components with different geometries can be effectively distinguished.

\section{Methodology}

2.1. ${ }^{252}$ Cf Sources-Driven Correlation Measurement Method. In ${ }^{252} \mathrm{Cf}$ sources-driven correlation measurement method, ${ }^{252} \mathrm{Cf}$ is employed as an interrogating neutron source. ${ }^{252} \mathrm{Cf}$ source, uranium component, neutron detectors, and relevant data acquisition and display system are included in the 


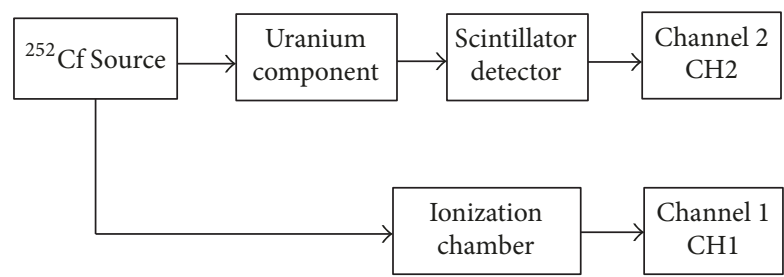

FIGURE 1: Flowchart of the proposed identification system.

measurement setup. Figure 1 shows the flowchart of the verification system. What if the uranium component is irradiated by ${ }^{252} \mathrm{Cf}$ source, ${ }^{235} \mathrm{U}$ goes through fission readily within $10^{-14}$ seconds in average and emits gamma rays, neutrons, and photons [19]. The mutual effects between spontaneous fission neutrons and gamma rays from ${ }^{252} \mathrm{Cf}$ source and the uranium component are chiefly in three possible processes: penetration, scattering, and induced fission. Then the neutrons and gamma rays are detected and recorded by the detector. This method takes advantage of innumerable neutron counts and makes inferences about the properties of uranium components observed by the correlation function between the ${ }^{252} \mathrm{Cf}$ source and detector.

2.2. The Correlation Function. As shown in Figure $1,{ }^{252} \mathrm{Cf}$ source is placed in an ionization chamber called channel 1 (CH1). The chamber's operation mode is typically pulse mode so that individual source fission event is observable. Neutrons from the ${ }^{252} \mathrm{Cf}$ source interacting with the uranium component are detected by a scintillator detector, which is placed straightly behind the uranium component and called channel $2(\mathrm{CH} 2)$. The uranium component is placed between the ${ }^{252} \mathrm{Cf}$ source and the scintillator detector.

Thus, we can obtain signals $X_{1}(t), X_{2}(t+\tau)$ from CH1 and $\mathrm{CH} 2$, respectively, and derive the correlation function between the two signals. The correlation function $C_{12}(\tau)$, which statistically reflects the correlation neutrons characteristics between two random signals, is defined as

$$
C_{12}(\tau)=\lim _{T \rightarrow \infty} \frac{1}{2 T} \int_{-T}^{T} X_{1}(t) X_{2}(t+\tau) \mathrm{d} t
$$

where $\tau$ is the delayed time and $T$ is the observing time. The correlation functions can depict some properties of uranium components, such as enrichment and mass.

Figure 2 shows the correlation function between the ${ }^{252} \mathrm{Cf}$ source and a detector measured by ORNL [22]. Figure 2 has several meaningful features marking as letters from (a) to (g). (a) denotes the emitting time of ${ }^{252} \mathrm{Cf}$ spontaneous fission. (b) shows the directly transmitted gamma rays recorded by the detector. (c) means the scattered gamma rays and directly transmitted neutrons. (d) corresponds to the scattered neutrons, the neutrons, and gamma rays from induced fission that are detected lately (e). Some of these divisions are not distinct in time but overlap. For a long time, slow decay (f) will reduce to the background level (g).

2.3. PCA-LSSVM. Principal component analysis (PCA) is a powerful multivariate statistical technique which usually

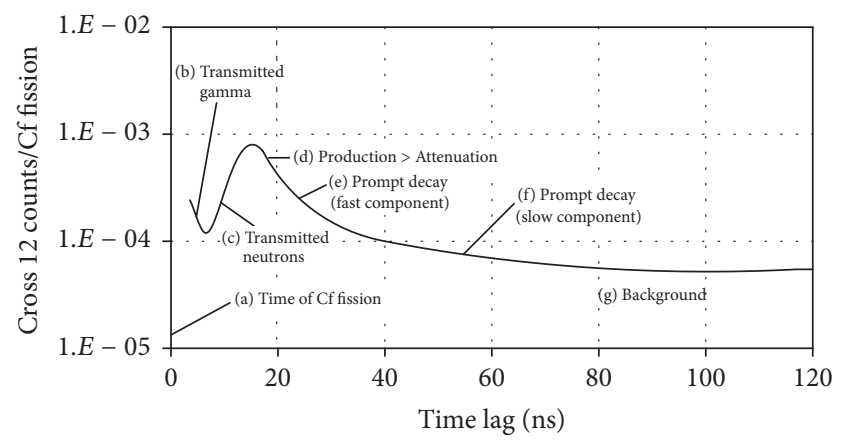

FIgURE 2: Correlation function between ${ }^{252} \mathrm{Cf}$ source and a detector $(\tau>0)$.

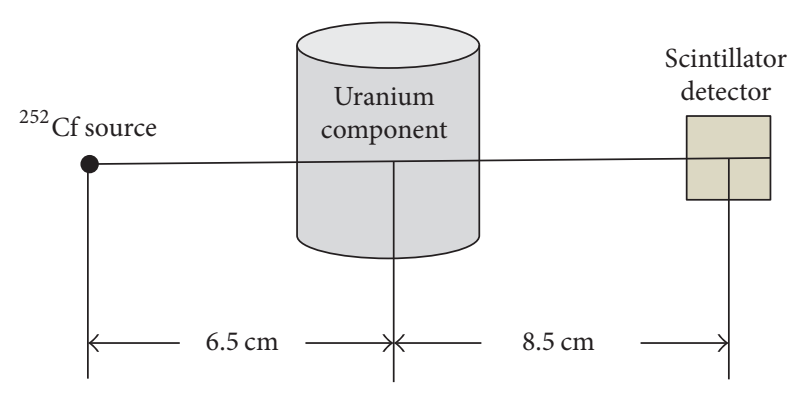

FIgURE 3: Structure of the simulation setup.

applied to data treatment of high dimensionality. It is based on the reduction of the variable numbers for a lower value of the p-original ones, in order to represent the characteristics of this data set, however, without loss of information. Therefore, it can cover the combination among the variables as well as grouping of samples. Least squares-support vector machine (LS-SVM) is a method based on the Statistical Learning Theory (SLT) that employs a least-square linear system as a cost function, resembling a regularized network. PCALSSVM deals with combination of the feature extraction and classification. Geometry identification of the uranium components is separated into two phases: (1) the feature extraction from the correlation functions and feature reduction by PCA and (2) the classification by LSSVM classifier.

\section{Simulations, Results, and Discussion}

3.1. Simulation Setup and Results. Considering high radioactivity of high-enrichment uranium components, the simulation method is commonly used in the first place instead of making some experiments. In fact, the simulation research will help us further improve the performance of real-data experiments. Correlation measurements were carried out by application of MC simulation. Figure 3 shows the structure of the simulation setup. The distance between the ${ }^{252} \mathrm{Cf}$ source and the detector is fixed for all measurements.

The particle transport code, Geant 4, was used for all simulations. Figure 4 shows the correlation functions acquired by the MC simulation. In order to make the results more accurate, we remove the transmitted gamma rays by our proposed "rejecting window" method [23, 24]. When 
TABLE 1: Parameters of the 3 uranium components.

\begin{tabular}{lccr}
\hline Geometry & Dimension & Volume & Surface area \\
\hline Sphere & Radius: $6 \mathrm{~cm}$ & $9.05 \times 10^{2} \mathrm{~cm}^{3}$ & $452.39 \mathrm{~cm}^{2}$ \\
\hline Cylinder & $\begin{array}{l}\text { Radius: } 6 \mathrm{~cm} \\
\text { Height: } 8 \mathrm{~cm}\end{array}$ & $9.05 \times 10^{2} \mathrm{~cm}^{3}$ & $527.79 \mathrm{~cm}^{2}$ \\
\hline \multirow{2}{*}{ Cuboid } & $\begin{array}{l}\text { Length: } 10 \mathrm{~cm} \\
\text { Width: } 10 \mathrm{~cm} \\
\text { Height: } 9.05 \mathrm{~cm}\end{array}$ & $9.05 \times 10^{2} \mathrm{~cm}^{3}$ & $561.91 \mathrm{~cm}^{2}$ \\
\hline
\end{tabular}

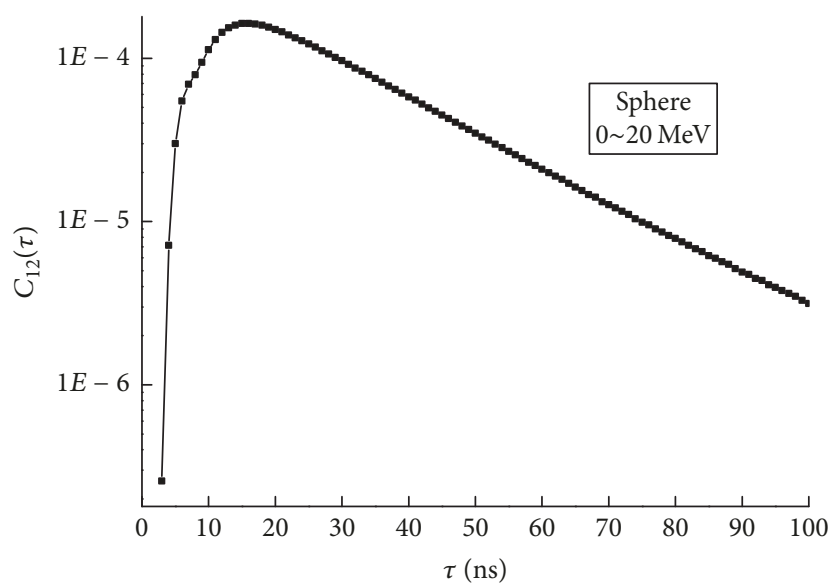

FIGURE 4: Simulation results of the sphere uranium component.

${ }^{252} \mathrm{Cf}$ spontaneous fission, directly transmitted neutrons, the scattered neutrons, and the neutrons from induced fission all are detected by the detector, the peaks appeared in the curve.

For the neutrons detected by our detectors, we choose part of them whose energy covers $0 \sim 20 \mathrm{MeV}$ as the objects to be processed. The average energy of ${ }^{252} \mathrm{Cf}$ source spontaneous fission neutron is $2.13 \mathrm{MeV}$. The average energy for the induced neutrons is about $2 \mathrm{MeV}$. Furthermore, neutron energy decreases when neutrons collide with uranium nuclei, which is beneficial to improving the probability of ${ }^{235} \mathrm{U}$ fission reaction. Thus, neutrons with energy less than $2 \mathrm{MeV}$ account for the vast majority. Neutrons with energy ranging $2 \sim 20 \mathrm{MeV}$ are very few. Neutrons with energy larger than $20 \mathrm{MeV}$ are rare. That is why we choose $0 \sim 20 \mathrm{MeV}$.

Compared with Figures 2 and 4, there are several differences: (1) due to eliminating the transmitted gamma rays, the feature (b) does not exist in Figure 4; (2) the experiment setups are different, such as the mass and enrichment of the uranium component. But beyond that, both results are consistent qualitatively in the total correlation functions. Thus it can be seen that the MC code is skilled in correctly simulating the correlation experiments between the ${ }^{252} \mathrm{Cf}$ source and detector.

3.2. Geometry Identification Based on Correlation Measurements. Three kinds of uranium components with the same mass $(16.9736 \mathrm{~kg})$ and same enrichment $(93.15 \%)$ but different geometry are used. Table 1 lists the parameters of 3 samples.
Figure 5 shows the correlation functions for the 3 uranium components in different energy intervals. Here we focus on neutron correlation events and remove the gamma rays firstly; there is no gamma peak in Figure 5. The directly transmitted neutrons are the first particles detected by $\mathrm{CH} 2$ and then the scattered neutrons and the fission neutrons induced from the uranium components. The correlation function distribution presents a wide range because neutrons move at different velocities. The distribution curves given in Figure 5 show that, as time goes on, the correlation function decreases with the neutron chain reaction progress. Considering the features of the correlation function and compared with the distribution curves after the neutron peak, Figure 5 shows inconsistently in different energy intervals and cannot realize effectively the geometry identification.

To analyze the parameters of 3 uranium components, which have the same enrichment, mass and volume, so they have the same neutron net production. The 3 samples have different geometry, so they have different superficial area. Different superficial areas lead to different neutron leakages. The bigger superficial area has more possibility of stimulating the heavy nuclei and to induce easily more fission. Considering the above, the geometry identification of the 3 samples is very difficult, because there are subtle differences in neutron signals. Even worse, the correlation functions include "real" coincidence and "accidental" coincidence as shown in Figure 6. It is worth noting in Figure 6 that the "accidental" coincidence is chiefly from scatter neutrons which is not conductive to the geometry identification. Unfortunately, the detector just records the neutron counts but cannot pick out the fission neutrons or scatter neutrons. So, the geometry identification is very difficult by the correlation functions simply.

3.3. Geometry Identification Model Based on PCA-LSSVM. In order to identify the geometry of uranium components efficiently, we proposed a method combined principal component analysis and least-square support vector machine (PCA-LSSVM) to build an identification model based on the correlation functions of the 3 different geometry properties. In the above research, the correlation functions were divided into different groups with an energy interval of $0.1 \mathrm{MeV}$. The sample number of each geometry property was 21 . So $21 \times 3$ $=63$ groups of correlation functions were obtained. The 63 groups of correlation functions were shown in Figure 7.

In Figure 7, with the increase of the energy, the correlation functions of the sphere in a high energy level were mixed with the correlation functions of the cylinder in a low level. 


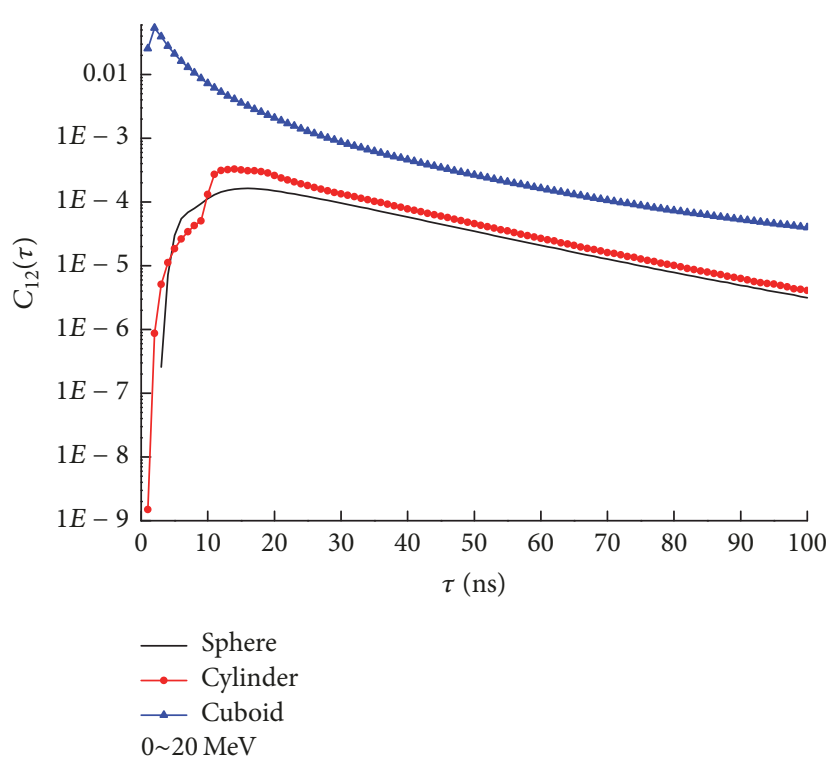

(a)

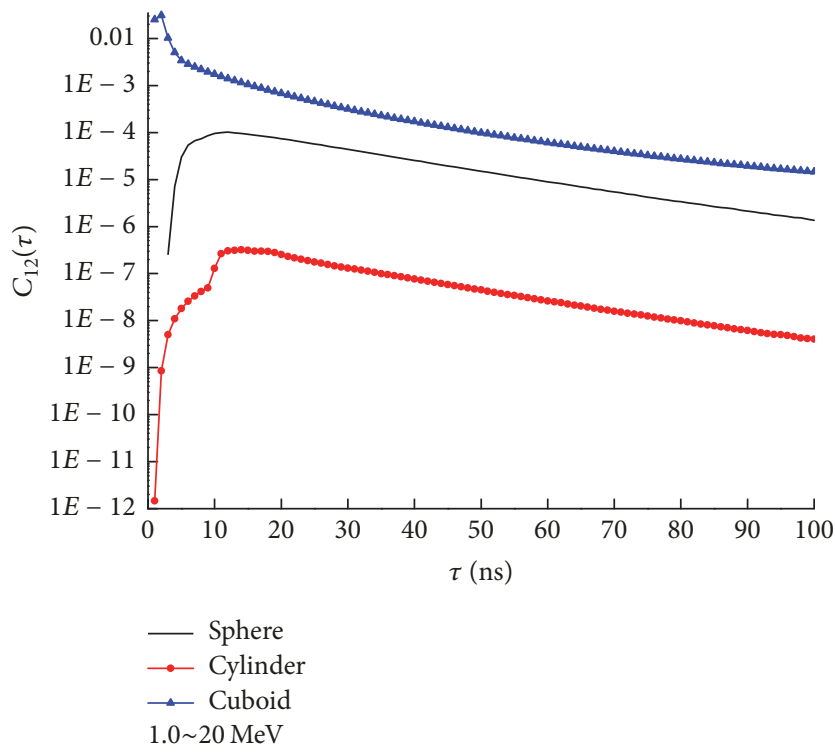

(c)

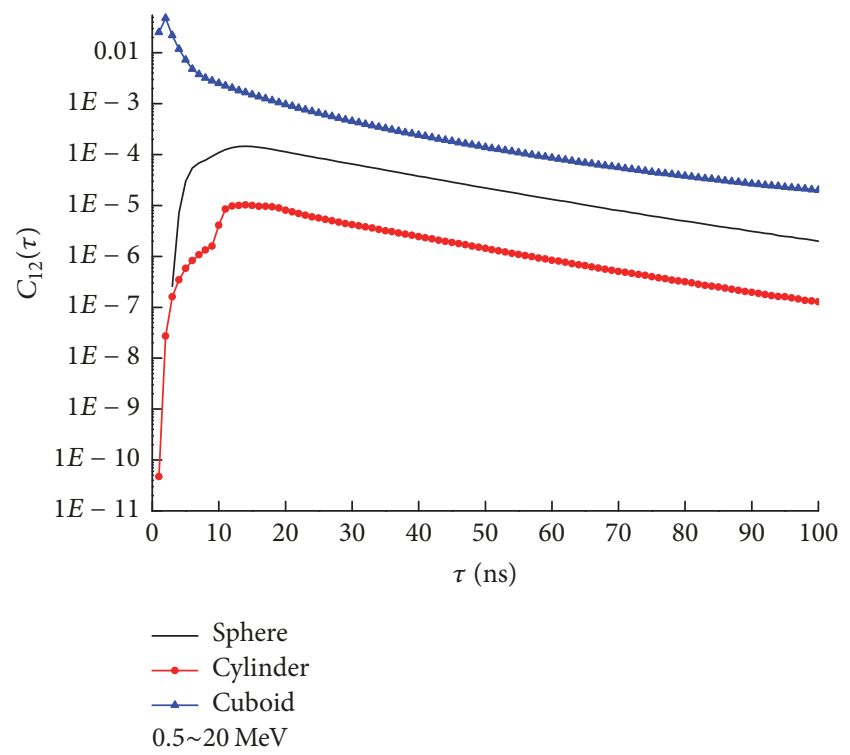

(b)

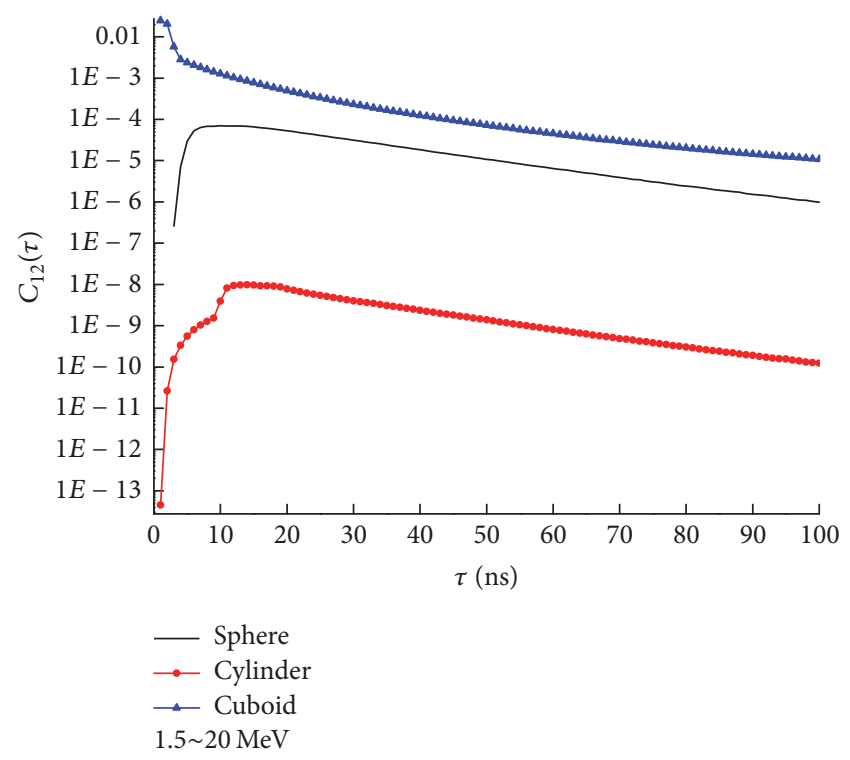

(d)

FIGURE 5: Simulation results of neutron correlation function.

The similar situation also existed in the correlation functions of cylinder and cuboid, which is consistent with the above results. The geometry property was unable to be identified directly with the correlation functions.

A geometry identification model based on PCA-LSSVM was constructed. Firstly, PCA was applied to extract the feature components from the 63 groups of correlation functions. The feature components were the linear combination of the correlation functions and they were able to characterize the original data well. According to the extraction through PCA, the redundant information was deleted and the dimension was reduced dramatically compared with the original data. The number of the feature components was related to the including information percentage. Normally, an information percentage of no less than $85 \%$ contained in feature components was acceptable. In the experiment, the information percentage was selected as $95 \%$ and 3 feature components were obtained. The coefficients of the first feature component were shown in Figure 8.

The coefficients of the first feature component were the weights in characterizing the geometry properties with the correlation functions. When building the identification model of the geometry properties, the feature components were applied to replace the correlation functions as the input of the identification model. The labels of the 3 geometry properties were sets 1,2 , and 3 as output of the model corresponding to sphere, cylinder, and cuboid, respectively. Then LSSVM was applied to train the identification model 


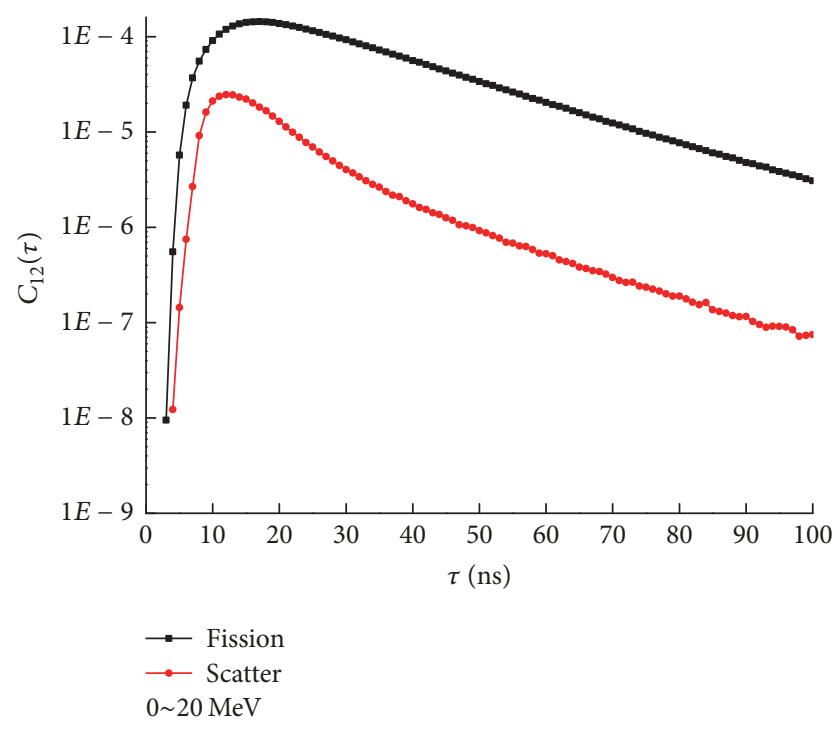

FIGURE 6: Simulation results of "real" and "accidental" coincidence.

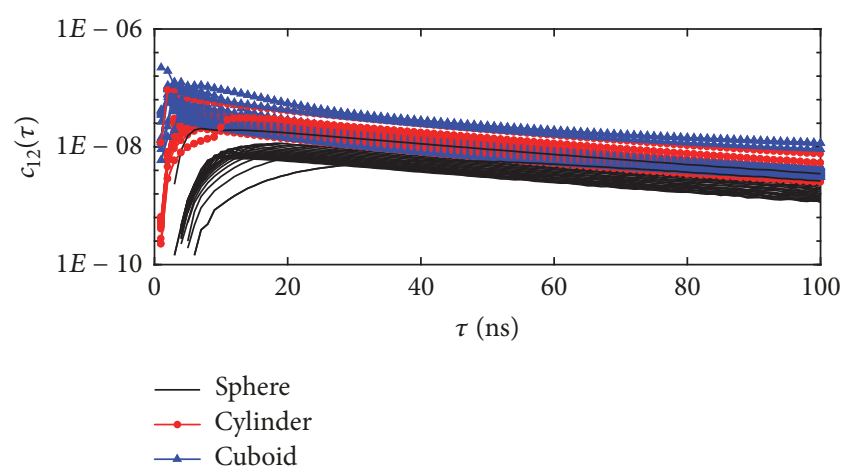

FIGURE 7: 63 groups of correlation functions of 3 different geometry properties.

of the geometry properties. In the model training process, the correlation functions were divided into training set and test set with a proportion of $13: 8$. Radial basis function was selected as the kernel function. The regularization parameter $c$ and kernel width $\sigma^{2}$ of the LSSVM were determined by particle swarm optimization (PSO) method. At last, the identification model was trained and shown in Figure 9. In Figure 9, the distributions of each sample in the principal component feature space were shown. Coordinate axis $X_{1}$ showed the value of first feature component of each sample while the $X_{2}$ showed the second. The identification model divided the space into three parts. Each part represented the region where the feature components of the corresponding geometry property distributed.

The training precision was $100 \%$ and the test precision was $95.83 \%$ of the identification model. The total precision of the identification model was $98.41 \%$, which indicated that the identification model was an effective way to identify the geometry properties with the correlation functions. However, the number of the sample data shown in this paper was not rich enough. In the future work, more data is expected to be achieved to test the reliability and precision of the

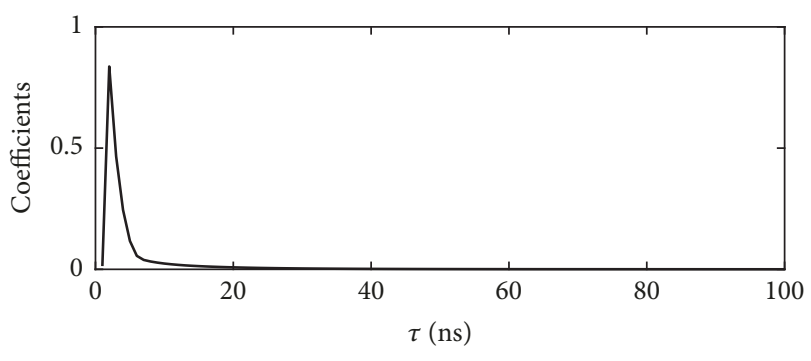

FIGURE 8: Coefficients of the first feature component.

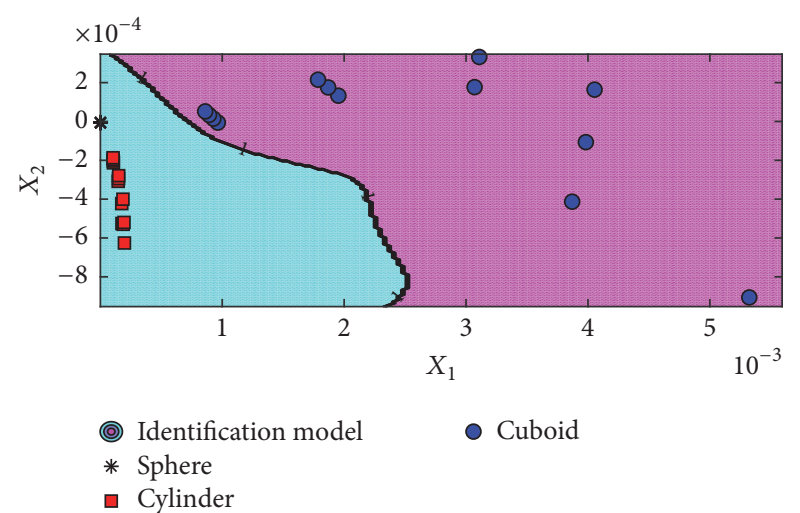

FIGURE 9: Identification model of the geometry properties trained by LSSVM.

identification model. Moreover, the identification model can be optimized for the possibility of identifying the geometry properties with different uranium masses and enrichments.

\section{Conclusions}

This paper describes a set of simulation experiments for the 3 uranium samples which have the the same mass and enrichment but different geometry. The experiments were performed using ${ }^{252} \mathrm{Cf}$ sources-driven correlation measurement method, based on the correlation functions. However, the correlation functions are disturbed by "accidental" coincidence chiefly from scatter neutrons and showed disagreement in different energy intervals. Therefore, we cannot realize the geometry identification by the correlation functions directly. We proposed further a method combined principal component analysis and least-square support vector machine (PCA-LSSVM). The results based on PCA-LSSVM showed that the training precision was $100 \%$ and the test precision was $95.83 \%$ of the identification model. The total precision of the identification model was $98.41 \%$, which indicated that the identification model was an effective way to identify the geometry properties with the correlation functions. Obviously, this correlation function based identification method should be feasible not only for these three geometries but also for other geometries; it is one of our follow-up studies.

\section{Conflicts of Interest}

The authors declare that they have no conflicts of interest. 


\section{Acknowledgments}

This work was partially supported by the National Natural Science Foundation of China (no. 11605017) and the Fundamental Research Funds for the Central Universities (no. 10611CDJXZ238826).

\section{References}

[1] X. W. Du, An elementary, of nuclear arms control technology, National Defense Industry Press, Beijing, China, 1996.

[2] C. A. Liu and J. Wu, An introduction of verification technology of nuclear arms control, vol. 3, National Defense Industry Press, Beijing, China, 2007.

[3] T. Uckan, M. Wyatt, J. Mihalczo, T. Valentine, J. Mullens, and T. Hannon, " ${ }^{252}$ Cf-source-correlated transmission measurements for uranyl fluoride deposit in a 24-in-OD process pipe," Nuclear Instruments and Methods in Physics Research, vol. 422, no. 1-3, pp. 26-34, 1999.

[4] J. L. Muñoz-Cobo, R. B. Perez, T. E. Valentine, Y. Rugama, and J. T. Mihalczo, "Stochastic transport theory of neutron and photon coupled fields: neutron and photon counting statistics in nuclear assemblies," Annals of Nuclear Energy, vol. 27, no. 12, pp. 1087-1114, 2000.

[5] T. Valentine and J. Mihalczo, "MCNP-DSP: A neutron and gamma ray Monte carlo calculation of source-driven noisemeasured parameters," Annals of Nuclear Energy, vol. 23, no. 16, pp. 1271-1287, 1996.

[6] G. P. Marc, M. Monterial, P. Marleau et al., "Gamma/neutron time-correlation for special nuclear material detection - Active stimulation of highly enriched uranium," Annals of Nuclear Energy, vol. 72, pp. 358-366, 2014.

[7] S. A. Pozzi and J. Segovia, " ${ }^{252}$ Cf source-correlated transmission measurements and genetic programming for nuclear safeguards," Nuclear Instruments and Methods in Physics Research A, vol. 491, no. 1-2, pp. 326-341, 2002.

[8] M. Marseguerra, E. Padovani, and S. A. Pozzi, "Use of the MCNP-Polimi code for time-correlation safeguards measurements," Progress in Nuclear Energy, vol. 43, no. 1-4, pp. 305-311, 2003.

[9] P. Feng, S. Y. Liu, and B. Wei, "Simulation and experimental study of a random neutron analyzing system with Cf- 252 neutron source," Nuclear Science and Techniques, vol. 22, pp. 39-46, 2011.

[10] J. T. Mihalczo and V. K. Paré, “Theory of correlation measurement in time and frequency domains with ${ }^{252} \mathrm{Cf}$," Annals of Nuclear Energy, vol. 2, no. 2-5, pp. 97-105, 1975.

[11] J. A. Williams, J. T. Mihalczo, C. W. Ricker, F. L. Glesius, and T. A. Kniss, "A high-sensitivity, position-sensitive fission chamber for subcriticality measurements of spent fuel," Nuclear Instruments and Methods in Physics Research Section A: Accelerators, Spectrometers, Detectors and Associated Equipment, vol. 299, no. 1-3, pp. 187-190, 1990.

[12] A. M. Stolle, "Application of stochastic diffusion theory to the interpretation of the ${ }^{252 C} \mathrm{f}$ source-driven noise analysis method for subcriticality determination," Annals of Nuclear Energy, vol. 20, no. 3, pp. 167-183, 1993.

[13] J. S. Li, Y. ZHang, and Y. Jin, "Development of ${ }^{252} \mathrm{Cf}$ fast fission chamber," Nuclear Electronics \& Detection Technlogy, vol. 21, no. 4, pp. 264-267, 2001.

[14] S. A. Pozzi, R. B. Oberer, L. G. Chiang, J. K. Mattingly, and J. T. Mihalczo, "Higher-order statistics from NMIS to measure neutron and gamma ray cross talk in plastic scintillators," Nuclear Instruments and Methods in Physics Research Section A, vol. 481, no. 1-3, pp. 739-748, 2002.

[15] B. R. Grogan and J. T. Mihalczo, "Simulating NMIS imaging and fission-mapping measurements," INMM 52nd Annual Meeting, 2011.

[16] J. Mihalczo, J. Mattingly, J. Neal, and J. Mullens, "NMIS plus gamma spectroscopy for attributes of HEU, PU and HE detection," Nuclear Instruments and Methods in Physics Research Section B, vol. 213, pp. 378-384, 2004.

[17] S. Zhang and J. Wu, "Active method of delayed neutrons to determine uranium enrichment," Nuclear Techniques, vol. 32, no. 12, pp. 922-926, 2009.

[18] P. Huang, J. S. Li, and et al., "Influence of uranium components geometry on time correlation coincidence mass measurement," Atomic Energy Science Technology, vol. 46, pp. 53-56, 2012.

[19] W. Xie, J. Li, J. Gong, J. Zhu, and P. Huang, "Experimental study on the measurement of uranium casting enrichment by timedependent coincidence method," Chinese Physics C, vol. 37, no. 10, 2013.

[20] Y. Zhang, Y. Qin, Z.-Y. Xing, L.-M. Jia, and X.-Q. Cheng, "Roller bearing safety region estimation and state identification based on LMD-PCA-LSSVM," Measurement, vol. 46, no. 3, pp. 13151324, 2013.

[21] D. Çalişir and E. Dogantekin, "A new intelligent hepatitis diagnosis system: PCA-LSSVM," Expert Systems with Applications, vol. 38, no. 8, pp. 10705-10708, 2011.

[22] J. T. Mihalczo, J. A. Mullens, J. K. Mattingly, and T. E. Valentine, "Physical description of nuclear materials identification system (NMIS) signatures," Nuclear Instruments and Methods in Physics Research Section A: Accelerators, Spectrometers, Detectors and Associated Equipment, vol. 450, no. 2, pp. 531-555, 2000.

[23] Y. Ren, B. Wei, J. Li, C. Ye, and P. Feng, "Implementation of ${ }^{252} \mathrm{Cf}$-source-driven power spectrum density measurement system," High Power Laser and Particle Beams, vol. 24, no. 1, pp. 215-219, 2012 (Chinese).

[24] B. Wei, J. Jin, Y. Ren, and Y. Tang, "Method of $\gamma$-peak removal in coincidence measurement system for verification of nuclear arms control," High Power Laser and Particle Beams, vol. 23, no. 10, pp. 2781-2785, 2011 (Chinese). 

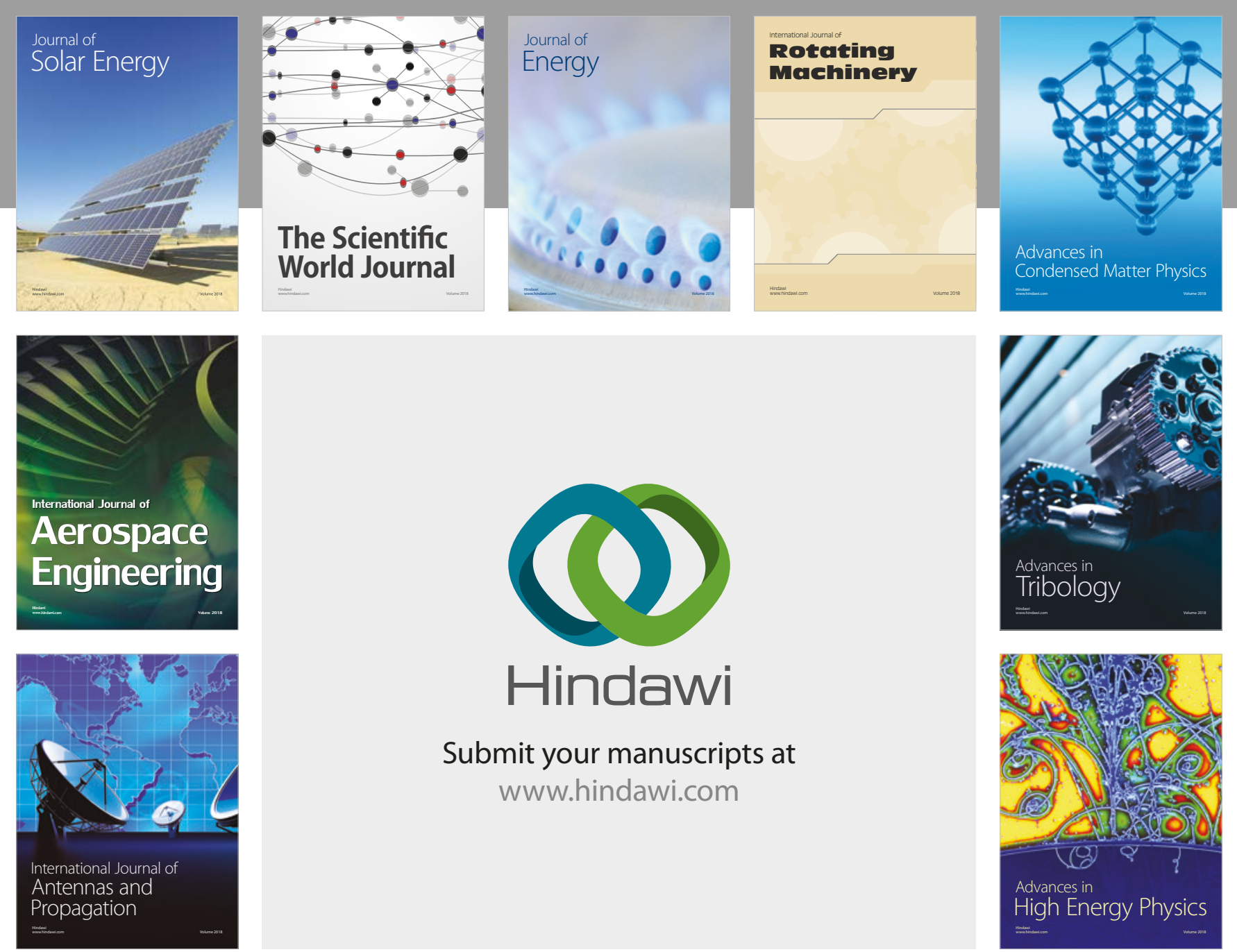

Submit your manuscripts at

www.hindawi.com
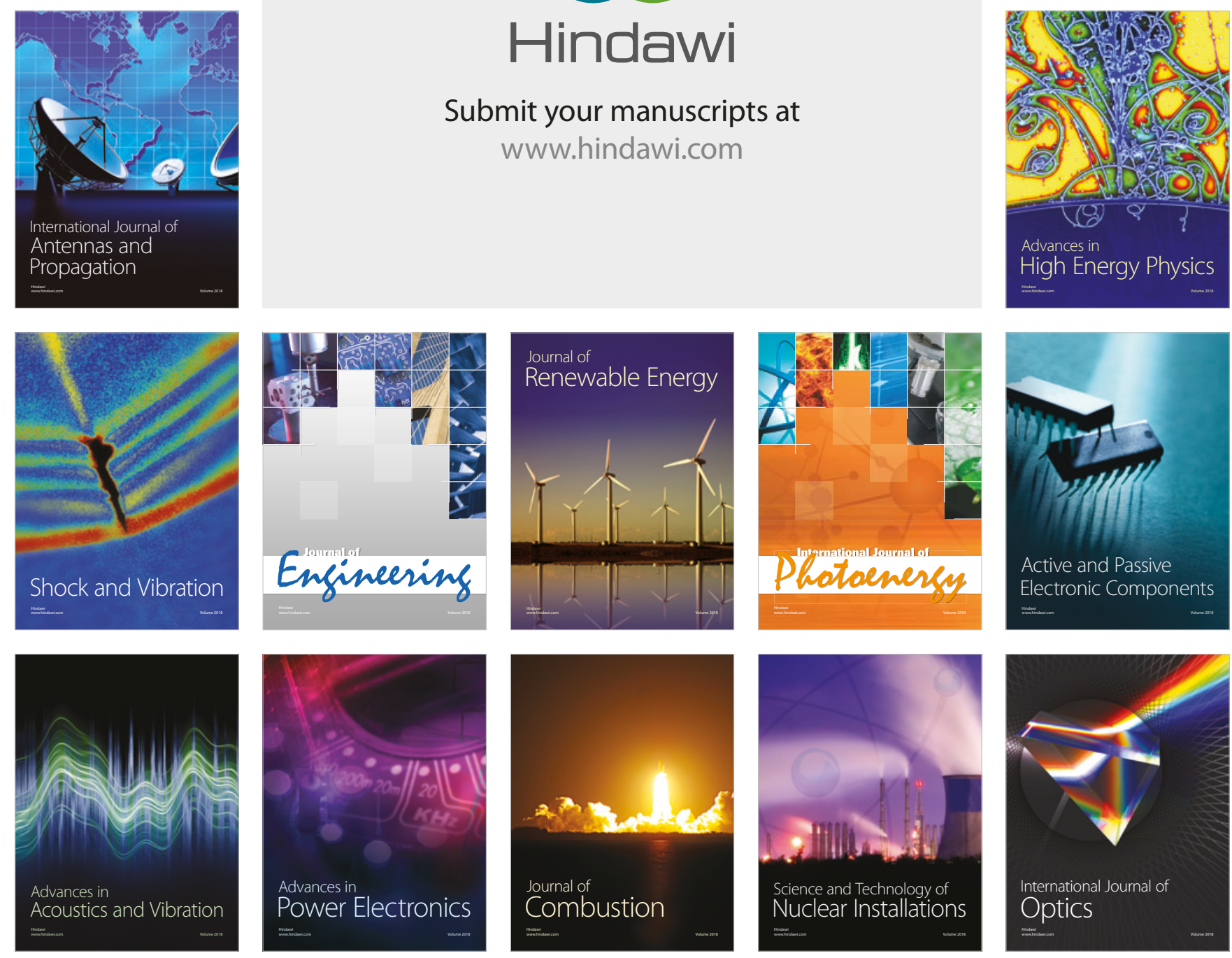